\title{
Augmented Telepresence Using Autopilot Airship and Omni-directional Camera
}

\author{
Fumio Okura* $\quad$ Masayuki Kanbara* Naokazu Yokoya*
}

Nara Institute of Science and Technology (NAIST)

\begin{abstract}
This study is concerned with a large-scale telepresence system based on remote control of mobile robot or aerial vehicle. The proposed system provides a user with not only view of remote site but also related information by AR technique. Such systems are referred to as augmented telepresence in this paper. Aerial imagery can capture a wider area at once than image capturing from the ground. However, it is difficult for a user to change position and direction of viewpoint freely because of the difficulty in remote control and limitation of hardware. To overcome these problems, the proposed system uses an autopilot airship to support changing user's viewpoint and employs an omni-directional camera for changing viewing direction easily. This paper describes hardware configuration for aerial imagery, an approach for overlaying virtual objects, and automatic control of the airship, as well as experimental results using a prototype system.

Index Terms: H.5.1 [Information Interfaces and Presentation]: Multimedia Information Systems-Artificial, augmented, and virtual realities I.2.9 [Artificial Intelligence]: Robotics-Autonomous vehicles
\end{abstract}

\section{INTRODUCTION}

This paper proposes a telepresence system which can superimpose virtual objects on a scene of remote site with AR technique. The telepresence system with augmented reality is referred to as augmented telepresence in this paper. In related work of the augmented telepresence, additional information used for teleoperation is overlaid on images captured by a mobile robot on the ground [1]. Another work of augmented telepresence uses unmanned aerial vehicle (UAV). Aerial images captured from radio controlled helicopter are used for augmented telepresence system [2]. Augmented telepresence systems using aerial images can superimpose much information on the image than systems using ground images because aerial imagery can observe a wider area. Such systems are expected to be used for not only teleoperation but also overlaying annotation to buildings or geographical information from the sky.

However, it is difficult to change position of user's viewpoint in augmented telepresence from the sky because remote control of UAV should be concerned with a number of factors: changing speed, turning left or right, changing altitude, and some environmental factors such as status of wind. In addition, if a standard camera is employed for observing the real scene, rotation of the vehicle is required to change user's viewing direction. It is usually difficult to get an arbitrary viewing direction in real-time.

In the proposed system, an autopilot airship supports a user to see an augmented scene which the user want to look around. The autopilot airship limits degrees of freedom (DOF) of remote control so that the user can control the vehicle simply. In addition, the proposed system equips an omni-directional camera which can capture a nearly full spherical view to enable the user to look around the scene without rotation of the vehicle.

*e-mail: \{fumio-o, kanbara, yokoya $\} @$ is.naist.jp

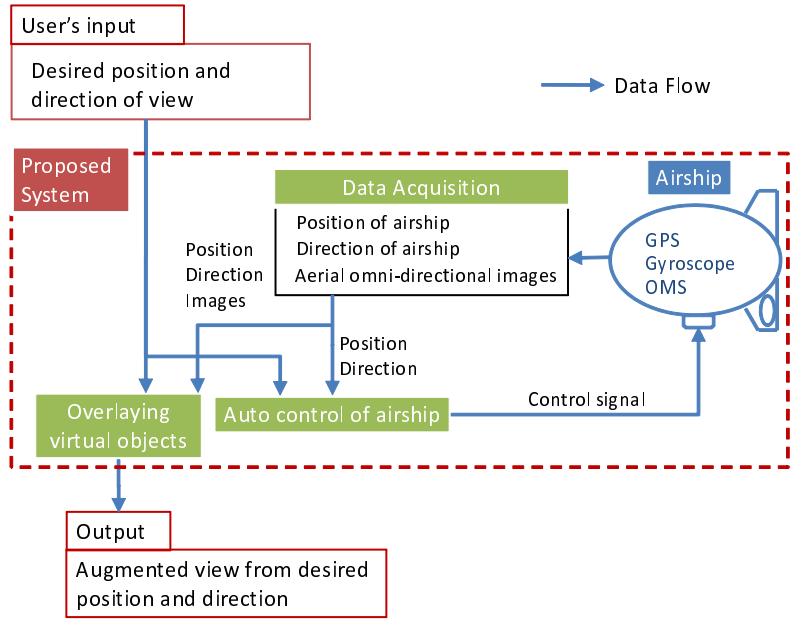

Figure 1: Data flow of proposed system

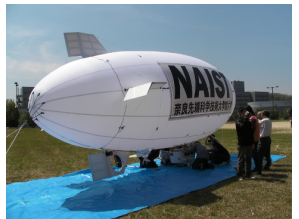

Figure 2: Unmanned airship

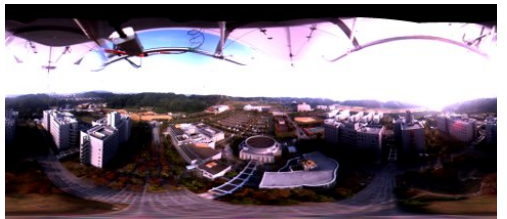

Figure 3: Aerial panoramic image captured by omni-directional camera

\section{System Overview}

Figure 1 illustrates data flow of the proposed system. User should input position and direction of a desired view. The system displays an augmented view from the position and direction. For automatic control and overlaying virtual object, a GPS and a gyroscope are equipped with the airship.

The proposed system consists of the following three processes.

1. Data acquisition

2. Overlaying virtual objects

3. Automatic control of airship

Detail of each process is described below.

\subsection{Data Acquisition}

Omni-directional aerial image is captured by an omni-directional multi camera system (OMS) equipped on an unmanned airship shown in Figure 2. Position and direction of the airship are measured by a GPS and a gyroscope. Figure 3 shows a panoramic image generated by using intrinsic and extrinsic parameters of each camera of the OMS [3].

\subsection{Overlaying virtual objects}

To generate an augmented image, the panoramic image captured by the OMS is projected onto a virtual spherical surface which has a sufficiently large radius centered at the camera, and is converted to a common planar-perspective image in the desired viewing direction. Virtual objects are overlaid on the perspective image by using position and direction of the OMS. 

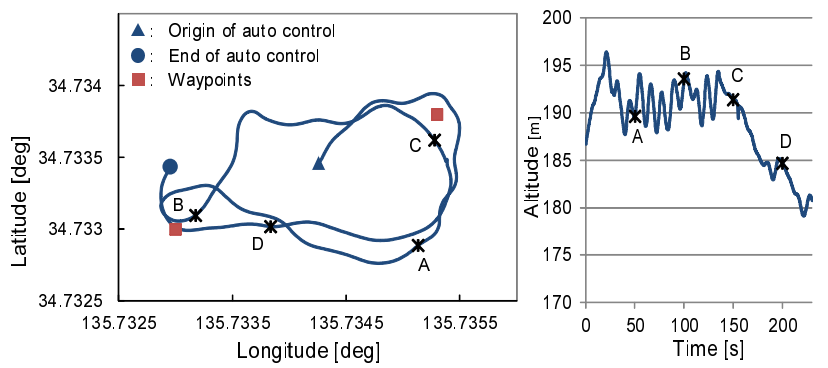

Figure 4: Trajectory of autopilot airship (Left: ground projection, Right: altitude): ' $*$ ' in the figure indicates positions of airship every 50 seconds.

Position and direction of the OMS are determined by the extrinsic camera parameter estimation method [4] based on structurefrom-motion and GPS data. At present, real-time camera parameter estimation is not implemented for on-line telepresence. In extrinsic camera parameter estimation using GPS data in large-scale environment, feature tracking often fails mainly due to error of the GPS. To overcome the problem, a long video sequence is divided into short sequences with hundreds of frames. Neighboring sequences are overlapped, and estimated camera parameters on overlapped frames are integrated by weighted averaging. Direction information from gyroscope is used as initial direction of the OMS in each sequence.

\subsection{Automatic control of airship}

To support changing user's viewpoint efficiently, the proposed system provides user with the following two different approaches to control airship.

\section{Following waypoints designated by user}

2. Reducing DOF of remote control

Each approach is detailed below.

\subsubsection{Following waypoints designated by user}

In this approach, the airship is controlled to follow the waypoints using a proportional control scheme [5]. The points can be designated in advance or on the fly. This approach is suitable when the desired viewpoint is clearly defined.

\subsubsection{Reducing DOF of remote control}

If desired position of viewpoint is not clear, it is required to control the airship to desired direction from current position. In usual remote control, the user should consider three DOF: speed, rudder, and elevation. This approach reduces DOF so as to control only speed and rudder, changing elevation automatically by using the proportional method [5].

\section{EXPERIMENTS}

\subsection{Prototype system}

To evaluate the proposed augmented telepresence system, we have carried out two experiments using a prototype system:

- Automatic control of airship by designating points in advance,

- Overlaying a virtual object on stored images captured from the airship.

Our unmanned airship shown in Figure 2 was used for a prototype system. The airship equips an OMS (Point Grey Research, Ladybug3), a differential-GPS (Hitachi Zosen Corporation, P4-GPS), and a fiber-optic gyroscope (Tokyo Keiki, TISS-5-40). All sensors were connected to a laptop PC on the airship to store and transmit captured data. Another PC placed on the ground was used to generate control signal for the airship. A transmitter was connected to the $\mathrm{PC}$ on the ground to transmit control signal to the airship.
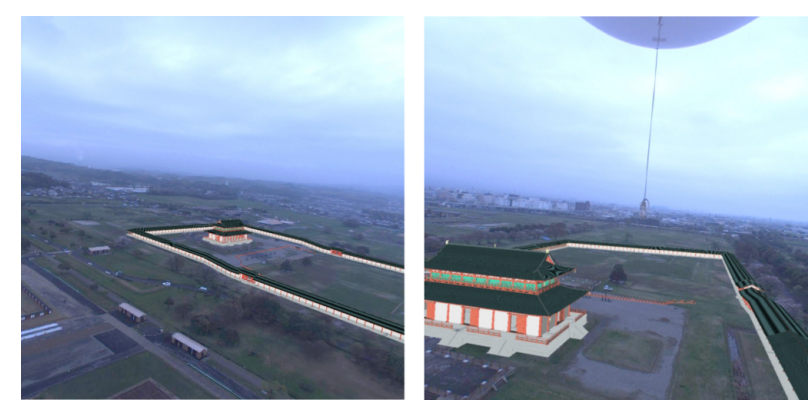

Figure 5: Examples of user's view from different viewpoints

\subsection{Automatic control of airship}

In the experiment about automatic control, the airship was controlled around two points $(230 \mathrm{~m}$ distance, $70 \mathrm{~m}$ altitude from the ground). The airship was controlled manually during takeoff and landing. Position and direction of the airship and control signal were generated in $4 \mathrm{~Hz}$. Figure 4 illustrates a trajectory of the autopilot airship. For the most part, the airship was controlled around the points. However, sometimes the airship drifted from the points mainly due to measurement error of the gyroscope.

\subsection{Overlaying a virtual object}

Virtual objects were overlaid on omni-directional images in offline processing because of the difficulty of omni-directional image transmission in the present system configration. The airship was controlled manually during capturing aerial images. In the experiment, a 3D model of an old palace in Heijo-capital was overlaid on a defunct base of the palace captured by the prototype system. The 3D model was successfully overlaid at appropriate positions in varying user's views as shown in Figure 5.

\section{CONCLUSION AND FUTURE WORK}

This paper has proposed a large-scale augmented telepresence system using aerial images captured from unmanned airship. The autopilot airship supports to change position of user's viewpoint, and an omni-directional camera realizes to change user's viewing direction without rotation of the airship. A GPS and a gyroscope are equipped on the airship to measure position and direction of the airship and the OMS. The experiments have shown that the airship can be controlled appropriately for most part, and a 3D model of an old palace is overlaid at appropriate position on a defunct base of the palace. In future work, real-time transmission of omni-directional images is required as well as real-time extrinsic camera parameter estimation to realize a real-time augmented telepresence system.

\section{ACKNOWLEDGEMENTS}

This work was supported in part by the "Ambient Intelligence" project granted by the Ministry of Education, Culture, Sports, Science and Technology.

\section{REFERENCES}

[1] S. Lawson, J. Pretlove, A. Weeler, and G. Parker. Augmented reality as a tool to aid the telerobotic exploration and characterization of remote environments. Presence, Vol. 2, No. 4, pp. 352-567, 2002.

[2] H. Kim, J. Kim, and S. Park. A bird's-eye view system using augmented reality. In Proc. of the Thirty-Second Annual Simulation Symposium, pp. 126-132, 1999.

[3] S. Ikeda, T. Sato, and N. Yokoya. High-resolution panoramic movie generation from video streams acquired by an omnidirectional multicamera system. In Proc. of IEEE Int. Conf. on Multisensor Fusion and Integration for Intelligent System (MFI2003), pp. 155-160, 2003.

[4] Y. Yokochi, S. Ikeda, T. Sato, and N. Yokoya. Extrinsic camera parameter estimation based-on feature tracking and GPS data. In Proc. of Asian Conf. on Computer Vision (ACCV2006), Vol. 1, pp. 369-378, 2006.

[5] E. Paiva, J. Azinheira, J. Ramos, A. Moutinho, and S. Bueno. Project AURORA: Infrastructure and flight control experiments for a robotic airship. J. of Field Robotics, Vol. 23, No. 2-3, pp. 201-222, 2006. 